Research Paper

\title{
P-selectin-mediated LOX expression promotes insulinoma growth in Rip1-Tag2 mice by increasing tissue stiffness
}

\author{
Cuiling $\mathrm{Qi}^{1}{ }^{1}$, , Jialin $\mathrm{Li}^{1}{ }^{1}$, Simei Guo ${ }^{1}$, Mengshi $\mathrm{Li}^{1}$, Yuanyuan $\mathrm{Li}^{3}$, Jiangchao $\mathrm{Li}^{1}$, Qianqian Zhang1, Lingyun

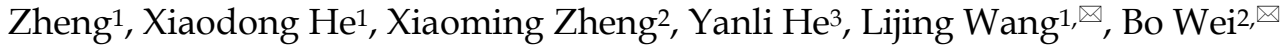 \\ 1. Vascular Biology Research Institute, School of Basic Course, Guangdong Pharmaceutical University, Guangzhou 510006, China \\ 2. Department of Gastrointestinal Surgery, the Third Affiliated Hospital, Sun Yat-sen University, Guangzhou, Guangdong 510630, China \\ 3. Department of Pathology, University of Guangzhou Chinese Medicine, Guangzhou, Guangdong 510000, China \\ * These authors contributed equally to this work. \\ $\square$ Corresponding authors: Bo Wei or Lijing Wang. Address: Department of Gastrointestinal Surgery, the Third Affiliated Hospital, Sun Yat-sen University, \\ Guangzhou, Guangdong 510630, P.R. China, or Vascular Biology Research Institute, School of Basic Course, Guangdong Pharmaceutical University, Guangzhou \\ 510006, P.R. China; Tel: +86-13527794069; E-mail: sanpi2013@163.com or wanglijing62@163.com
}

(C) Ivyspring International Publisher. Reproduction is permitted for personal, noncommercial use, provided that the article is in whole, unmodified, and properly cited. See http://ivyspring.com/terms for terms and conditions.

Received: 2016.06.07; Accepted: 2016.09.04; Published: 2016.10.18

\begin{abstract}
P-selectin, a cell adhesion molecule, is an important member of the selectin family. Recent studies have shown that P-selectin deletion inhibits tumor growth in Ripl-Tag2 mice by suppressing platelet accumulation in tumor tissues. This study aimed to evaluate whether and how P-selectin affects tumor stiffness in Rip1-Tag2 mice. To explore the role of P-selectin in tissue stiffness, we demonstrated that tumor progression in Ripl-Tag2 mice was correlated with tissue stiffness using immunofluorescence and histological staining. Furthermore, we showed that P-selectin deficiency significantly decreased tissue stiffness by inhibiting lysyl oxidase (LOX) expression. Our experiments involving Rip 1-Tag2 mice treated with the LOX inhibitor BAPN showed that BAPN significantly abolished collagen deposition to decrease tumor stiffness and thus inhibit tumor growth. These results indicate that P-selectin deletion significantly decreases tumor stiffness in Ripl-Tag2 mice by inhibiting LOX expression. Further study demonstrated that P-selectin-mediated platelet accumulation increases tissue stiffness mainly by increasing LOX expression and thus promotes tumor growth. Therefore, P-selectin may be an effective therapeutic targeting for treating human insulinomas.
\end{abstract}

Key words: P-selectin, insulinoma, tissue stiffness, LOX, Rip1-Tag2 mice

\section{Introduction}

P-selectin (CD62P), a cell adhesion molecule, is an important member of the selectin (CD62) family. Many studies have reported that P-selectin has significant effects on the growth and metastasis of many cancers including melanoma, lung cancer, and colorectal carcinoma. Furthermore, our previous study demonstrated that P-selectin deletion inhibited melanoma metastasis by suppressing platelet adhesion and that P-selectin-mediated platelet adhesion promoted intestinal tumor growth in $\mathrm{Apc}^{\mathrm{Min} /+}$ mice $[1,2]$. Apc $\mathrm{Min} /+$ mice are a spontaneous intestinal adenomatous polyps model, as they contain an Apc gene mutation that causes multiple intestinal neoplasia (Min). Importantly, we found that P-selectin deficiency inhibited insulinoma progression using Rip1-Tag2 transgenic mice and Rip1-Tag2;P-sel-/mice [3]. We also found that large numbers of platelets accumulated in Rip1-Tag2 mouse insulinomas via a P-selectin-dependent mechanism [3]. Rip1-Tag2 mice are a spontaneous insulinoma model resulting from the expression of the SV40 T antigen, which functions as an oncogene, in $\beta$-cells [4]. Tumor development in Rip1-Tag2 mice involves a multistep process including a hyperplastic islet stage, an angiogenic islet stage, a tumor stage and an invasive cancer stage. Angiogenic islets appear in 
six-week-old Rip1-Tag2 mice, and some of these angiogenic islets develop into tumors in nine-week-old Rip1-Tag2 mice [4]. However, the relationships between P-selectin and P-selectin-mediated platelet aggregation and insulinoma ECM stiffness remain unclear.

Some studies have shown that many types of tumors are stiffer than adjacent tissues and that tumor ECM stiffness is strongly correlated with solid tumor progression and aggression [5,6]. Collagen is the most abundant component of tumor extracellular matrix (ECM) [7]. Evidence from various studies regarding human cancers and experimental tumor models indicates that cross-linked collagen accumulation can enhance tumor ECM stiffness. Acerbi et al reported that human breast and epithelial cancer progression is facilitated by collagen deposition and remodeling, which are closely associated with tumor stiffness [8-10]. However, the relationship between tumor stiffness and insulinoma development and progression remains unknown.

Lysyl oxidase (LOX), a secreted copper-dependent amine oxidase, is a collagen cross-linker [11]. Studies have demonstrated that LOX is highly expressed in tumor cells and that LOX inhibitors can inhibit tumor progression by decreasing the amount of collagen cross-links in tumors [12]. Breast cancer patients exhibiting high levels of LOX expression have a greater chance of developing metastasis and thus experience shorter survival times than patients exhibiting low levels of LOX expression [13-17]. LOX-mediated cross-linking and increases in collagen concentrations can heighten collagen stiffness, indicating that ECM stiffness promotes breast cancer growth and metastasis [18] and that the extent of collagen deposition and the amount of collagen cross-links in tumors control tumor progression [19].

In this study, we sought to investigate the relationship between P-selectin expression and insulinoma ECM stiffness and the role of P-selectin-mediated ECM stiffness in insulinoma growth in Rip1-Tag2 mice. We found that collagen deposition increased with tumor progression in Rip1-Tag2 mice and Rip1-Tag2;P-sel-/- mice. Our previous study also demonstrated that P-selectin deletion inhibit insulinoma growth in Rip1-Tag2 mice [9]. Furthermore, we investigated the relationship between P-selectin expression and insulinoma ECM stiffness using Rip1-Tag2 mice. Our findings indicated that P-selectin deletion decrease insulinoma ECM stiffness during every stage of insulinoma progression in Rip1-Tag2 mice, thereby inhibiting insulinoma growth in these mice. More importantly, we demonstrated that the LOX inhibitor BAPN suppressed insulinoma growth in Rip1-Tag2 mice by reversing P-selectin-mediated increases in insulinoma ECM stiffness. We previously demonstrated that P-selectin promotes platelet accumulation in insulinomas in Rip1-Tag2 mice, thereby promoting insulinoma growth [3]. We also found that P-selectin-mediated platelet accumulation promotes increases in tissue stiffness mainly by increasing LOX expression.

\section{Materials and Methods}

\section{Mice}

P-selectin knockout (P-sel-/-) mice and Rip1-Tag2 transgenic mice were purchased from the Jackson Laboratory (the Jackson Laboratory, Bar Harbor, ME, USA) and the National Cancer Institute (NCI, Washington, DC, USA). Male Rip1-Tag2 mice were crossed with female $\mathrm{P}$-sel $\%$ mice to establish Rip1-Tag2;P-sel- $/$ mice. The genotypes of the Rip1-Tag2 mice and $\mathrm{P}$-sel-/- mice were identified as previously described [9]. The animals were housed under specific pathogen-free conditions, and all experiments were performed in accordance with institutional guidelines. The animal protocol was approved by the Medical Research Animal Ethics Committee of Guangdong Pharmaceutical University.

\section{Immunofluorescence staining}

Pancreases from Rip1-Tag2 mice and Rip1-Tag2;P-sel $\%$ mice were fixed in $4 \%$ paraformaldehyde overnight and then embedded in optimum cutting temperature compound (OCT) and sectioned. For immunofluorescence staining, the sections were rehydrated in distilled water, blocked with $10 \%$ bovine serum albumin (BSA) and incubated with a primary antibody against collagen type I (Abcam, Cambridge, CB, UK) overnight at $4{ }^{\circ} \mathrm{C}$. The next day, DyLight 488 or 555-conjugated anti-GPIb $\beta$ antibodies (Invitrogen, Carlsbad, CA, USA) were added to the sections, which were counterstained with 4'-6-diamidino-2-phenylindole (DAPI). For immunofluorescence staining quantitation, we randomly chose fields from at least five tumors per mouse (typically 10-15) in five to eight mice per group using a $40 \times$ objective lens. The slides were collected, and the images were quantified using Image-Pro Plus software (IPP, version 6.0, Media Cybernetics).

\section{Histopathological staining}

Pancreases from Rip1-Tag2 mice and Rip1-Tag2;P-sel $/-$ mice were fixed in $10 \%$ formalin and then embedded with paraffin and sectioned. The tissue sections $(3 \mu \mathrm{m})$ were stained with $\mathrm{H} \& \mathrm{E}$, Masson's trichrome and reticulin using reagents and kits from Maxim-Bio (Maxim-Bio, Fuzhou, Fujian, 

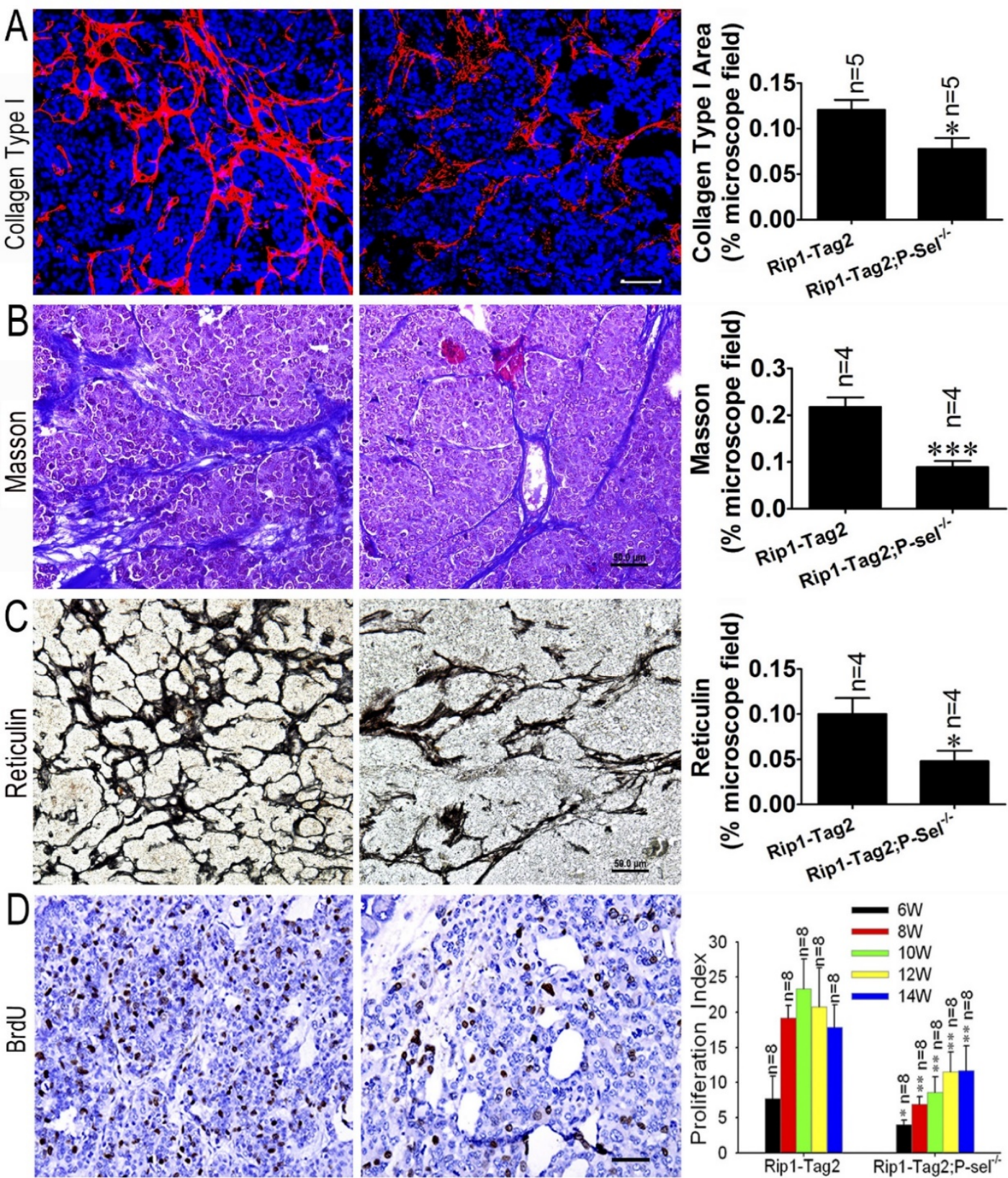

Rip1-Tag2

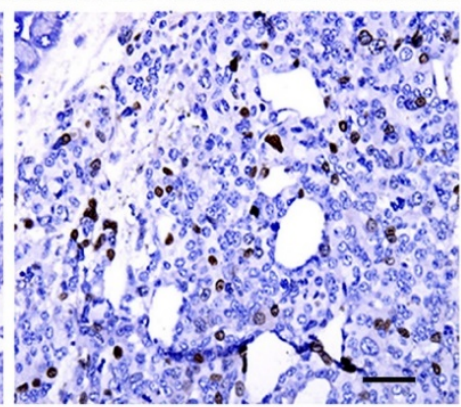

Rip1-Tag2; P-sel-

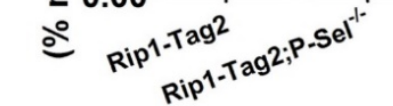

Figure 2. P-selectin deletion decreases tissue stiffness in Rip1-Tag2 mouse insulinomas. (A) Immunofluorescence staining showing collagen type I deposition in the insulinomas of 12-week-old Rip1-Tag2 and Rip1-Tag2;P-sel-/- mice. Rip1-Tag2;P-sel-/- mice exhibited less collagen type I deposition than Rip1-Tag2 mice. (B) Representative images and quantitation of Masson's staining of insulinoma sections from 12-week-old Rip1-Tag2 and Ripl-Tag2;P-sel ${ }^{-/-}$mice. Collagen fiber deposition levels were determined by evaluating the blue areas as percentages of the total microscope area. (C) Representative images and quantitation of reticulin staining of insulinoma sections from 12-week-old Rip1-Tag2 and Rip1-Tag2;P-sel-/- mice. Rip1-Tag2;P-sel-/- mice exhibited fewer reticular fibers than Rip1-Tag2 mice. (D) Insulinoma proliferative index. Proliferating cells were identified via immunohistochemical staining against BrdU antibodies. The numbers of positive cells per unit area were counted. At least ten sections from five mice were examined for statistical analysis. $*, P<0.05 ; * *, P<0.01$. Bar $=50 \mu \mathrm{m}$.

\section{P-selectin deficiency decreases tissue stiffness in Rip1-Tag2 mouse insulinomas}

We previously reported that P-selectin deletion significantly inhibits insulinoma growth in Rip1-Tag2 mice [9] and that tumor tissue stiffness increases in conjunction with insulinoma progression (Figure 1). To investigate the effects of P-selectin on insulinoma tissue stiffness, we subjected Rip1-Tag2 and
Rip1-Tag2;P-sel- $/$ mouse insulinomas to immunofluorescence, Masson's trichrome and reticulin staining for histological analysis. Immunofluorescence and Masson's trichrome staining showed that Rip1-Tag2;P-sel-/- mouse insulinomas exhibited a significantly decrease in collagen density (Figure 2A and 2B). Accordingly, reticulin staining showed that reticular fiber density was significantly decreased in Rip1-Tag2;P-sel ${ }^{-}$- 
mouse insulinomas compared to Rip1-Tag2 mouse insulinomas (Figure 2C). These results demonstrate that P-selectin deficiency decreases fiber deposition in Rip1-Tag2 mouse insulinomas and that fiber deposition is closely associated with tissue stiffness. Consistent with these findings, P-selectin deficiency suppressed tumor cell proliferation, as demonstrated via BrdU staining for proliferating cells (Figure 2D).

\section{P-selectin deficiency decreases hydroxyproline levels in Rip1-Tag2 mouse insulinomas}

Hydroxyproline, a component amino acid of collagen, is a parallel biochemical marker of fibrosis [22]. To investigate the role of P-selectin in insulinoma tissue stiffness further, we measured hydroxyproline levels in Rip1-Tag2 and Rip1-Tag2;P-sel ${ }^{-/}$mouse insulinomas. Rip1-Tag2;P-sel $\%$ mice exhibited significantly decreased-insulinoma hydroxyproline concentrations compared to control mice (Figure 3).

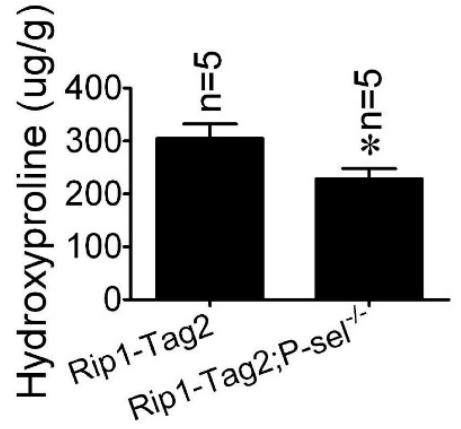

Figure 3. Hydroxyproline concentrations in the insulinomas of 12-week-old Ripl-Tag2 and Rip1-Tag2;P-sel-/- mice. Hydroxyproline levels were higher in the insulinomas of Ripl-Tag2 mice than in the insulinomas of Ripl-Tag2;P-sel-/- mice. *, $P<0.05$.

\section{The LOX inhibitor BAPN inhibits tumor growth in Rip1-Tag2 mice}

To determine how P-selectin affects insulinoma tissue stiffness in Rip1-Tag2 mice, we performed immunohistochemistrical staining to investigate LOX protein expression in the insulinomas of 12-week-old Rip1-Tag2 and Rip1-Tag2;P-sel-/- mice. LOX
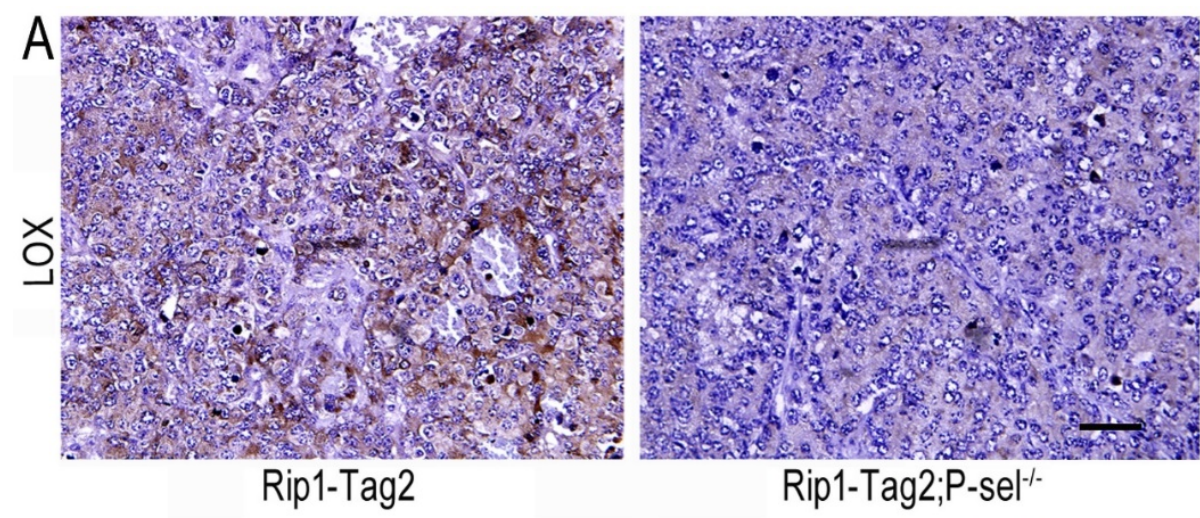
expression in Rip1-Tag2 mouse insulinomas was strong, while LOX expression in Rip1-Tag2;P-sel-/- mouse insulinomas was very weak (Figure 4A). Our results also demonstrated that LOX expression levels were significantly lower in Rip1-Tag2;P-sel-/- mouse insulinomas than in the Rip1-Tag2 mouse insulinomas (Figure 4B).
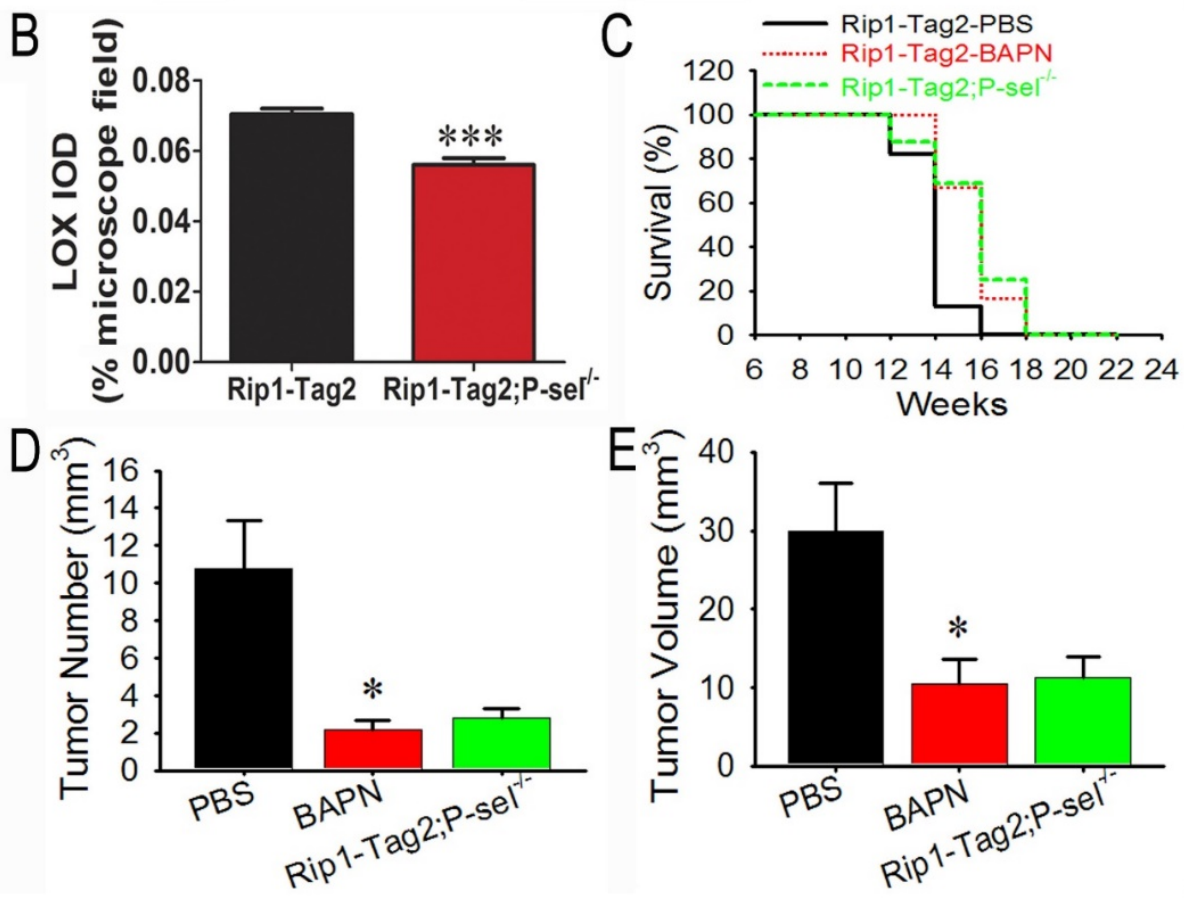

Figure 4. The effects of the LOX inhibitor BAPN on tumor growth in Rip1-Tag2 mice. (A and B) Immunohistochemical staining was performed to detect LOX expression in Rip1-Tag2 and Ripl-Tag2;P-sel ${ }^{-/}$mouse insulinomas. Representative images and semi-quantitation of LOX expression in the insulinomas of 12-week-old Rip1-Tag2 and Rip1-Tag2;P-sel ${ }^{-/}$mice. (C) The survival times of BAPN-treated Ripl-Tag2; P-sel-mice and Ripl-Tag2 mice were significantly prolonged compared to those of PBS-treated Rip1-Tag2 mice. (D and E) Tumor volumes (D) and numbers (E) were significantly decreased after BAPN treatment and P-selectin deletion. *, $P<$ $0.05 ; * * *, P<0.001$. Bar $=50 \mu \mathrm{m}$. 
Because LOX expression was down-regulated in Rip1-Tag2;P-sel $/$ - mouse insulinomas, the biological effects of LOX were investigated in the setting of tumor progression in Rip1-Tag2 mice. BAPN, a well-recognized inhibitor of LOX activity [23], was administered to Rip1-Tag2 mice. The survival rates of BAPN-treated Rip1-Tag2 and Rip1-Tag2;P-sel-/- mice were significantly higher than those of normal water-treated Rip1-Tag2 mice. Furthermore, there was no significant difference in survival time between BAPN-treated Rip1-Tag2 mice and Rip1-Tag2;P-sel-/mice (Figure 4C). Rip1-Tag2;P-sel $\%$ mice and BAPN-treated Rip1-Tag2 mice also exhibited fewer and smaller tumors than normal water-treated Rip1-Tag2 mice (Figure 4D and 4E). There were no differences in tumor numbers or volumes between BAPN-treated Rip1-Tag2 and Rip1-Tag2;P-sel-/- mice (Figure 4D and 4E). These results show that P-selectin deletion inhibits tumor progression in Rip1-Tag2 mice
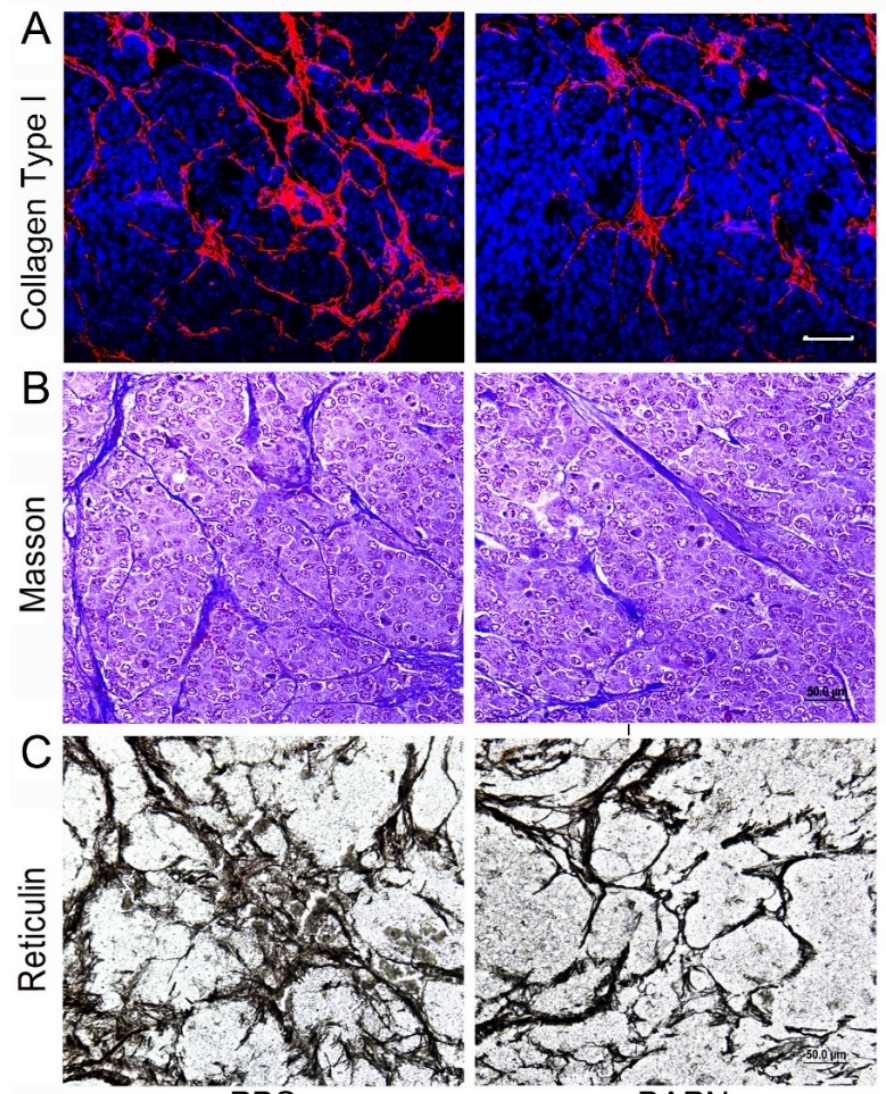

PBS

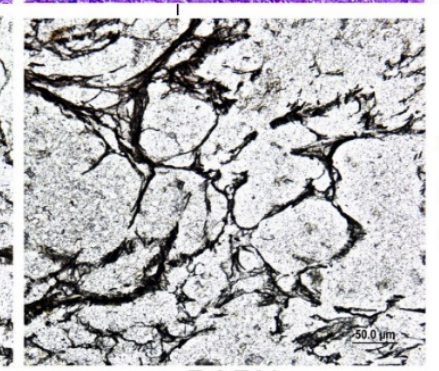

BAPN

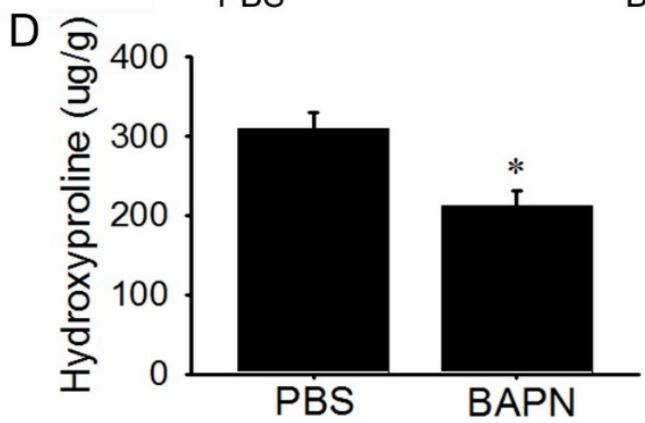

mainly by activating LOX.

\section{The LOX inhibitor BAPN decreases tissue stiffness inRip1-Tag2 mouse insulinomas}

To investigate the effects of the LOX inhibitor BAPN on insulinoma tissue stiffness, Rip1-Tag2 mouse insulinomas treated with BAPN and PBS were subjected to immunofluorescence, Masson's trichrome and reticulin staining for histological analysis. The immunofluorescence and Masson's trichrome staining results showed that collagen density was significantly decreased in Rip1-Tag2 mouse insulinomas treated with BAPN (Figure 5A and $5 \mathrm{~B}$ ). Furthermore, reticulin staining demonstrated that reticular fiber dentistry was significantly decreased in Rip1-Tag2 mouse insulinomas treated with BAPN compared to control mice (Figure 5C). To determine the role of P-selectin in insulinoma tissue stiffness, we measured hydroxyproline levels in Rip1-Tag2 mouse insulinomas treated with BAPN
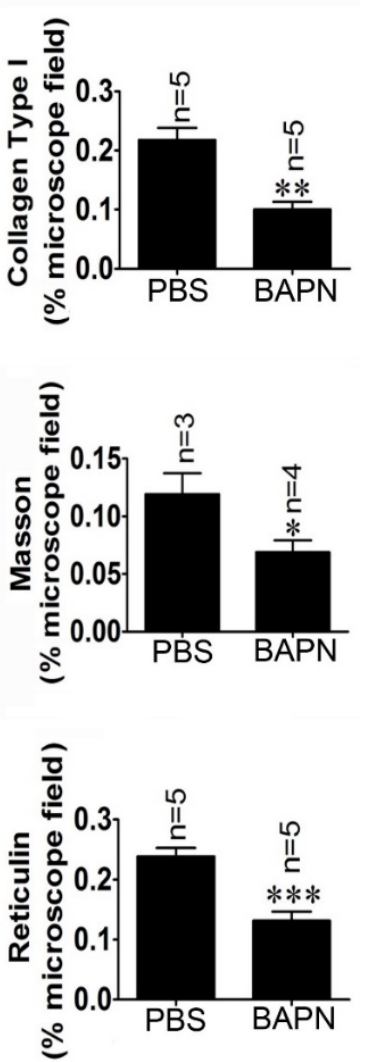
and PBS. Rip1-Tag2 mice treated with BAPN exhibited significantly decreased insulinoma hydroxyproline levels compared to control mice (Figure 5D). These results show that BAPN inhibits fiber deposition in Rip1-Tag2 mouse insulinomas and that fiber deposition is closely associated with tissue stiffness.

Figure 5. BAPN decreases tissue stiffness in Rip1-Tag2 mouse insulinomas. (A) The insulinoma sections of Rip1-Tag2 mice treated with normal water or BAPN water were subjected to immunofluorescence staining for collagen type I. Representative images and quantitation of immunofluorescence staining in the insulinoma sections of Rip1-Tag2 mice treated with BAPN or PBS. Ripl-Tag2 mice treated with BAPN exhibited much less type I collagen deposition than control mice. (B) Representative images and quantitation of Masson's staining of insulinoma sections from Rip1-Tag2 mice treated with BAPN or PBS. Collagen fiber deposition levels were determined by evaluating the blue areas as percentages of the total microscope area. (C) Representative images and quantitation of reticulin staining of insulinoma sections from Rip1-Tag2 mice treated with BAPN or PBS. Rip1-Tag2 mice treated with BAPN exhibited less reticular fiber deposition than control mice. (D) Hydroxyproline concentrations were detected in the insulinomas of 12-week-old Ripl-Tag2 mice treated with BAPN and PBS. Hydroxyproline levels were lower in the insulinomas of Rip1-Tag2 mice treated with BAPN than in the insulinomas of control mice. *, $P<0.05$; **, $P<0.01$; ***, $P<$ 0.001 . Bar $=50 \mu \mathrm{m}$. 


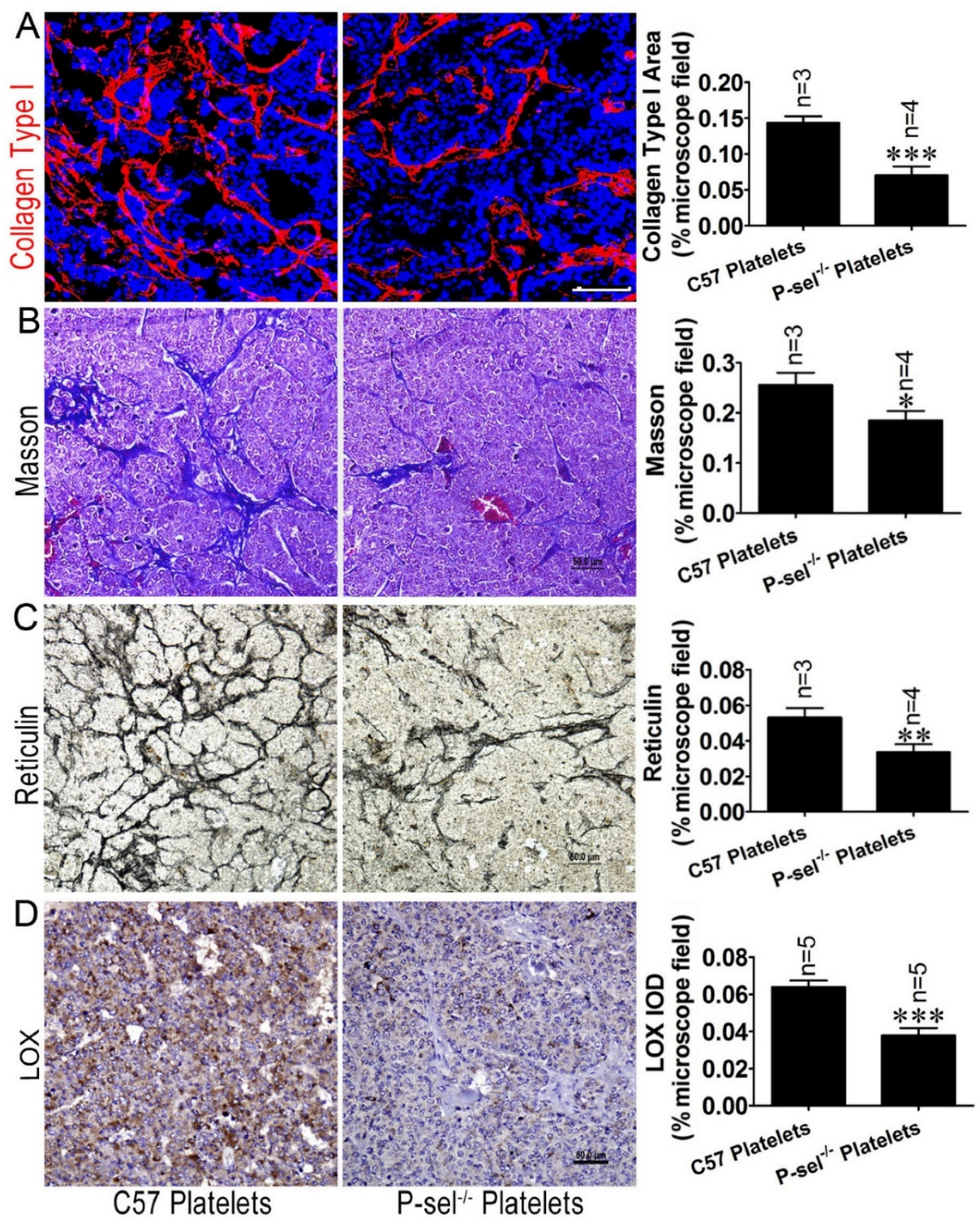

Figure 6. P-selectin-mediated platelet adhesion increases tissue stiffness by increasing LOX expression. (A) The insulinoma sections of Ripl-Tag2; $\mathrm{P}$-sel- - - mice treated with $\mathrm{C} 57$ platelets or $\mathrm{P}$-sel- ${ }^{-/-}$platelets were subjected to immunofluorescence staining for collagen type I. Representative images and quantitation of immunofluorescent staining of insulinoma sections from Ripl-Tag2;P-sel-/- mice treated with C57 platelets or P-sel ${ }^{-/}$platelets. Ripl-Tag2;P-sel ${ }^{-/-}$mice treated with P-sel ${ }^{-/}$platelets exhibited lower levels of collagen type I deposition than control mice. (B) Representative images and quantitation of Masson's staining of insulinoma sections from Ripl-Tag2;P-sel-/- mice treated with C57 platelets or P-sel- - platelets. Collagen fiber deposition levels were determined by evaluating the blue areas as percentages of the total microscope area. (C)

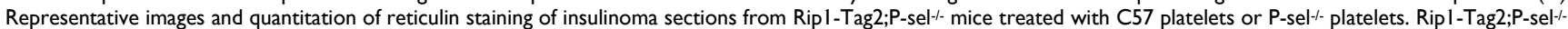
mice treated with P-sel-/- platelets exhibited less reticular fiber deposition than control mice. (D) Immunohistochemical staining was performed to detect LOX expression in the insulinomas of Ripl-Tag2; P-sel-/- mice treated with C57 platelets or P-sel ${ }^{-/-}$platelets. Representative image and semi-quantitation of LOX expression in the insulinomas of 12-week-old Rip1-Tag2;P-sel $/$ - mice treated with C57 platelets or P-sel ${ }^{-/-}$platelets.*, $P<0.05 ; * *, P<0.01 ; * * *, P<0.001$. Bar $=50 \mu \mathrm{m}$.

\section{P-selectin-mediated platelet adhesion increases tissue stiffness by increasing LOX expression}

To investigate the effects of P-selectin-mediated platelet adhesion on insulinoma tissue stiffness, Rip1-Tag2;P-sel $/-$ mice were treated with C57 and P-sel $/$ - platelets. Their insulinomas were subjected to immunofluorescence, Masson's trichrome and reticulin staining for histological analysis. The immunofluorescence and Masson's trichrome staining results demonstrated that collagen density was significantly decreased in the insulinomas of Rip1-Tag2 mice treated with $\mathrm{P}$-sel $/$ - platelets (Figure 6A and 6B). Reticulin staining showed that reticular fiber density was significantly decreased in the insulinomas of Rip1-Tag2 mice treated with $\mathrm{P}$-sel-/platelets compared to control mice (Figure 6C). 
Given that P-selectin-mediated platelet adhesion increases tissue stiffness, we elected to evaluate how P-selectin-mediated platelet adhesion affects tumor stiffness. We detected LOX protein expression in the insulinomas of Rip1-Tag2;P-sel $/$ - mice treated with C57 platelets and $\mathrm{P}$-sel $/$ - platelets via immunohistochemical staining. LOX expression was increased in the insulinomas of Rip1-Tag2;P-sel-/mice treated with C57 platelets, while LOX expression was weak in the insulinomas of Rip1-Tag2;P-sel-/mice treated with $\mathrm{P}^{-} \mathrm{sel}^{-/}$- platelets (Figure 6D). These results show that P-selectin-mediated platelet adhesion significantly affects fiber deposition, which is closely associated with tissue stiffness.

\section{Discussion}

It was previously reported by us that P-selectin deficiency can inhibit insulinoma growth in Rip1-Tag2 mice by suppressing platelet accumulation in tumor tissues [9]. Here, we showed that a correlation exists among ECM mechanical properties, P-selectin and insulinoma growth in Rip1-Tag2 mice. Our findings demonstrate that tumor progression in Rip1-Tag2 mice corresponds with significant increases in collagen deposition and linearization, which increase ECM stiffness. Importantly, P-selectin deletion significantly inhibits collagen deposition and linearization, thereby suppressing insulinoma growth in Rip1-Tag2 mice. The LOX inhibitor BAPN significantly suppresses insulinoma growth in Rip1-Tag2 mice, but not Rip1-Tag2;P-sel $\%$ mice, in conjunction with changes in ECM remodeling and stiffening. These results demonstrate that a correlation exists between tumor aggression and P-selectin-mediated increases in tumor stiffness in Rip1-Tag2 mice and that tumor stiffness is determined by collagen cross-links, especially the total amount of collagen cross-links.

Previous investigations have demonstrated that tissue stiffness contributes to tumor progression. P-selectin-mediated collagen deposition significantly increases ECM stiffness, which thereafter affects $\beta$-cell proliferation in Rip1-Tag2 mice (Figure 2), thereby promoting insulinoma growth in these mice. Our previous results indicated that P-selectin (an important member of the selectin family) deficiency suppresses melanoma metastasis and insulinoma growth by preventing platelet adhesion [7,9]. Furthermore, increases in tumor stiffness can exert diverse effects on tumor cell proliferation, differentiation, and migration and hence significantly modify tumor progression [24-27].

Increases in insulinoma ECM stiffness play an important role in insulinoma growth in Rip1-Tag2 and Rip1-Tag2;P-sel $/$ - mice. Increases in ECM stiffness are, at least in part, caused by increases in the total amount of collagen crosslinks [28]. Collagen crosslinking is induced predominantly by LOX and LOX-like (LOXLs) enzymes, which are synthesized by stromal cells during the early stages of cancer and synthesized by both stromal cells and cancer cells in response to hypoxia $[29,30]$. Furthermore, LOX is frequently highly expressed in many cancers [28], and we found that LOX expression levels were high in Rip1-Tag2 mouse insulinomas. P-selectin deletion significantly decreased HIF-1a and LOX expression in Rip1-Tag2 mouse insulinomas. In addition, we treated Rip1-Tag2 and Rip1-Tag2;P-sel-/- mice with BAPN, a LOX inhibitor, to determine the effects of LOX on insulinomas. BAPN treatment prevented collagen crosslinking and suppressed ECM stiffness in Rip1-Tag2 mice, thereby reducing tumor volumes and prolonging survival times in Rip1-Tag2 mice. However, in Rip1-Tag2;P-sel/- mice, BAPN treatment had no effect on tumor growth. Using a breast cancer mouse model, Levental KR et al also demonstrated that LOX inhibitor prevents tissue stiffening, thereby preventing breast cancer progression [28]. Our results indicate that P-selectin mediated-platelet adhesion induces LOX synthesis, which leads to collagen crosslinking.

In this study, we found that P-selectin mediated-platelet adhesion increases collagen deposition in Rip1-Tag2 mouse insulinomas by promoting LOX expression and thus increasing ECM stiffness. Increases in ECM stiffness promote insulinoma growth in Rip1-Tag2 mice. These findings indicate that ECM stiffness is associated with increased-insulinoma aggressiveness, which is mediated by P-selectin. Therefore, P-selectin may be an effective therapeutic targeting for treating human insulinoma.

\section{Acknowledgements}

This work was supported by grants from the National Science Foundation of China (31500966 to Cuiling Qi, 31471290 to Lijing Wang, and 81472825 to Bo Wei), the Science and Technology Planning Project of Guangdong Province (2014A020212315 and 2015A020211029 to Cuiling Qi and 2014B020212012 to Lijing Wang), the National Science Foundation of Guangdong (2014A030313078 to Bo Wei), the Young Innovative Talent Project of the Guangdong Province Education Department (2014KQNCX141 to Cuiling Qi), and the Science and Technology Planning Project of Guangzhou City (201607010135 to Cuiling Qi).

\section{Competing Interests}

The authors have declared that no competing 


\section{interest exists.}

\section{References}

1. Qi CL, Wei B, Ye J, Yang Y, Li B, Zhang QQ, et al. P-selectin-mediated platelet adhesion promotes the metastasis of murine melanoma cells. PLoS One. 2014;9:e91320.

2. Qi C, Li B, Guo S, Wei B, Shao C, Li J, et al. P-Selectin-Mediated Adhesion between Platelets and Tumor Cells Promotes Intestinal Tumorigenesis in ApcMin/+ Mice. Int J Biol Sci. 2015;11:679-87.

3. Qi C, Wei B, Zhou W, Yang Y, Li B, Guo S, Li J et al. P-selectin-mediated platelet adhesion promotes tumor growth. Oncotarget. 2015;6:6584-96.

4. Lopez T, Hanahan D. Elevated levels of IGF-1 receptor convey invasive and metastatic capability in a mouse model of pancreatic islet tumorigenesis. Cancer Cell. 2002;1:339-53.

5. Samani A, Bishop J, Luginbuhl C, Plewes DB. Measuring the elastic modulus of ex vivo small tissue samples. Phys Med Biol. 2003;48:2183-98.

6. Samani A, Zubovits J, Plewes D. Elastic moduli of normal and pathological human breast tissues: an inversion-technique-based investigation of 169 samples. Phys Med Biol. 2007;52:1565-76.

7. Kolacna L, Kolácná L, Bakesová J, Varga F, Kostáková E, Plánka L, Necas A, et al. Biochemical and biophysical aspects of collagen nanostructure in the extracellular matrix. Physiol Res. 2007;56:S51-S60.

8. Acerbi I, Cassereau L, Dean I, Shi Q, Au A, Park C, et al. Human Breast Cancer Invasion and Aggression Correlates with ECM Stiffening and Immune Cell Infiltration. Integr Biol (Camb). 2015;7:1120-34

9. Jodele S, Blavier L, Yoon JM, DeClerck YA. Modifying the soil to affect the seed: role of stromal-derived matrix metalloproteinases in cancer progression. Cancer Metastasis Rev. 2006;25:35-43.

10. Butcher DT, Alliston T, Weaver VM. A tense situation: forcing tumour progression. Nat Rev Cancer. 2009;9:108-122.

11. Yamauchi $M$, Sricholpech $M$. Lysine post-translational modifications of collagen. Essays Biochem. 2012;52:113-33.

12. Barker HE, Cox TR, Erler JT. The rationale for targeting the LOX family in cancer. Nat Rev Cancer. 2012;12:540-52.

13. Pickup MW, Laklai H, Acerbi I, Owens P, Gorska AE, Chytil A, et al. Stromally derived lysyl oxidase promotes metastasis of transforming growth factor- $\beta$-deficient mouse mammary carcinomas. Cancer Res. 2013;73:5336-46.

14. Erler JT, Weaver VM. Three-dimensional context regulation of metastasis. Clin Exp Metastasis. 2009;26:35-49.

15. Erler JT, Giaccia AJ. Lysyl oxidase mediates hypoxic control of metastasis. Cancer Res. 2006;66:10238-41.

16. Decitre M, Gleyzal C, Raccurt M, Peyrol S, Aubert-Foucher E, Csiszar K, et al. Lysyl oxidase-like protein localizes to sites of de novo fibrinogenesis in fibrosis and in the early stromal reaction of ductal breast carcinomas. Lab Invest. 1998;78:143-51.

17. Semenza GL. Cancer-stromal cell interactions mediated by hypoxia-inducible factors promote angiogenesis, lymphangiogenesis, and metastasis. Oncogene. 2013; 32:4057-63.

18. Dvorak HF, Weaver VM, Tlsty TD, Bergers G. Tumor microenvironment and progression. J Surg Oncol. 2011;103:468-74.

19. Chen Y, Terajima M, Yang Y, Sun L, Ahn YH, Pankova D, et al. Lysyl hydroxylase 2 induces a collagen cross-link switch in tumor stroma. J Clin Invest. 2015;125:1147-62.

20. Shi K, Zhao W, Chen Y, Ho WT, Yang P, Zhao ZJ. Cardiac hypertrophy associated with myeloproliferative neoplasms in JAK2V617F transgenic mice. J Hematol Oncol. 2014;7:25

21. Koczor CA, Torres RA, Fields E, Qin Q, Park J, Ludaway T, et al. Transgenic mouse model with deficient mitochondrial polymerase exhibits reduced state IV respiration and enhanced cardiac fibrosis. Lab Invest. 2013;93:151-8.

22. Ahmed LA, El-Maraghy SA, Rizk SM. Role of the KATP channel in the protective effect of nicorandil on cyclophosphamide-induced lung and testicular toxicity in rats. Sci Rep. 2015;5:14043.

23. Beerlage C, Greb J, Kretschmer D, Assaggaf M, Trackman PC, Hansmann ML, et al. Hypoxia-inducible factor 1-regulated lysyl oxidase is involved in Staphylococcus aureus abscess formation. Infect Immun. 2013;81:2562-73.

24. Paszek MJ, Weaver VM. The tension mounts: mechanics meets morphogenesis and malignancy. J Mammary Gl and Biol Neoplasia. 2004;9:325-42.

25. Paszek MJ, Zahir N, Johnson KR, Lakins JN, Rozenberg GI, Gefen A, et al. Tensional homeostasis and the malignant phenotype. Cancer Cell. 2005;8:241-54

26. Wozniak MA, Desai $\mathrm{R}$, Solski PA, Der CJ, Keely PJ. ROCK-generated contractility regulates breast epithelial cell differentiation in response to the physical properties of a three-dimensional collagen matrix. J Cell Biol. 2003;163:583-95.

27. Chun TH, Hotary KB, Sabeh F, Saltiel AR, Allen ED, Weiss SJ. A pericellular collagenase directs the 3-dimensional development of white adipose tissue. Cell. 2006;125:577-91.

28. Levental KR, Yu H, Kass L, Lakins JN, Egeblad M, Erler JT, et al. Matrix crosslinking forces tumor progression by enhancing integrin signaling. Cell. 2009;139:891-906.

29. Peyrol S, Raccurt M, Gerard F, Gleyzal C, Grimaud JA, Sommer P. Lysyl oxidase gene expression in the stromal reaction to in situ and invasive ductal breast carcinoma. Am J Pathol. 1997;150:497-507.
30. Santhanam AN, Baker AR, Hegamyer G, Kirschmann DA, Colburn NH Pdcd4 repression of lysyl oxidase inhibits hypoxia-induced breast cancer cell invasion. Oncogene. 2010;29:3921-32. 